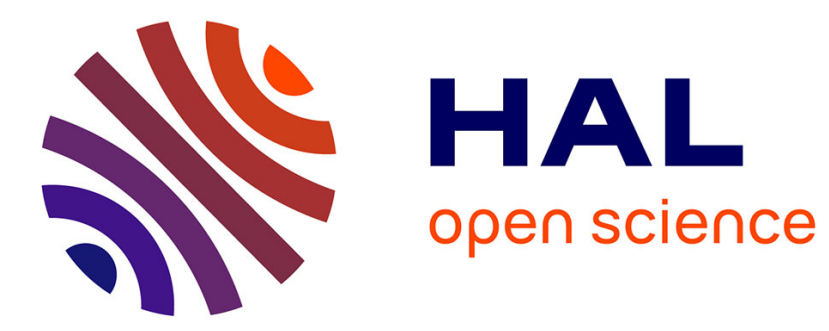

\title{
Convergent expressions for periodic potentials in stratified media using asymptotic extractions
}

Guido Valerio, Simone Paulotto, Paolo Baccarelli, David R. Jackson, Donald R. Wilton, William A. Johnson, Allessandro Galli

\section{- To cite this version:}

Guido Valerio, Simone Paulotto, Paolo Baccarelli, David R. Jackson, Donald R. Wilton, et al.. Convergent expressions for periodic potentials in stratified media using asymptotic extractions. IEEE Transactions on Magnetics, 2016, 52 (3), pp.7207804. 10.1109/TMAG.2015.2499087 . hal-01315756

\section{HAL Id: hal-01315756 https://hal.sorbonne-universite.fr/hal-01315756}

Submitted on 12 Mar 2020

HAL is a multi-disciplinary open access archive for the deposit and dissemination of scientific research documents, whether they are published or not. The documents may come from teaching and research institutions in France or abroad, or from public or private research centers.
L'archive ouverte pluridisciplinaire HAL, est destinée au dépôt et à la diffusion de documents scientifiques de niveau recherche, publiés ou non, émanant des établissements d'enseignement et de recherche français ou étrangers, des laboratoires publics ou privés. 


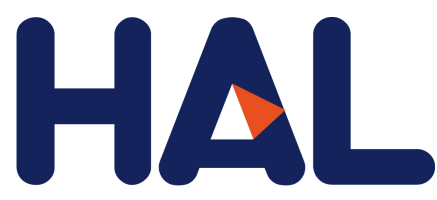

archives-ouvertes

\title{
Convergent expressions for periodic potentials in stratified media using asymptotic extractions
}

\author{
Guido Valerio, Simone Paulotto, Paolo Baccarelli, David Jackson, Donald \\ Wilton, William Johnson, Allessandro Galli
}

\section{To cite this version:}

Guido Valerio, Simone Paulotto, Paolo Baccarelli, David Jackson, Donald Wilton, et al.. Convergent expressions for periodic potentials in stratified media using asymptotic extractions. IEEE Transactions on Magnetics, Institute of Electrical and Electronics Engineers, 2016, 52 (3), pp.7207804. 10.1109/TMAG.2015.2499087 . hal-01315756

\section{HAL Id: hal-01315756 \\ https://hal.sorbonne-universite.fr/hal-01315756}

Submitted on 12 Mar 2020

HAL is a multi-disciplinary open access archive for the deposit and dissemination of scientific research documents, whether they are published or not. The documents may come from teaching and research institutions in France or abroad, or from public or private research centers.
L'archive ouverte pluridisciplinaire HAL, est destinée au dépôt et à la diffusion de documents scientifiques de niveau recherche, publiés ou non, émanant des établissements d'enseignement et de recherche français ou étrangers, des laboratoires publics ou privés. 


\title{
Convergent Expressions for Periodic Potentials in Stratified Media using Asymptotic Extractions
}

\author{
Guido Valerio ${ }^{1}$, Member, IEEE, Simone Paulotto ${ }^{2}$, Senior Member, IEEE, Paolo Baccarelli ${ }^{3}$, Member, IEEE, \\ David R. Jackson ${ }^{4}$, Fellow, IEEE, Donald R. Wilton ${ }^{4}$, Life Fellow, IEEE, \\ William A. Johnson ${ }^{5}$, Senior Member, IEEE, and Alessandro Galli ${ }^{3}$, Member, IEEE \\ ${ }^{1}$ Sorbonne Universités, UPMC Univ Paris 06, UR2, L2E, F-75005, Paris, France \\ ${ }^{2}$ Maxtena Inc., Bethesda, MD 20814 USA \\ ${ }^{3}$ Dpt. Information Engineering, Electronics and Telecommunications, Sapienza University of Rome, 00184, Rome, Italy \\ ${ }^{4}$ Dpt. Electrical and Computer Engineering, University of Houston, Houston, TX 77204-4005 USA \\ ${ }^{5}$ Electromagnetics consultant, Albuquerque, NM 87123-2421 USA
}

\begin{abstract}
The efficient computation of periodic Green's functions is discussed here for an arbitrarily directed array of point sources in layered media. These Green's functions are necessary to formulate boundary integral equations for arrays of scatterers inside a general layered medium, solved with a method of moments in the spatial domain. For this reason, mixed-potential Green's functions -having a mild spatial singularity-are selected. The case of horizontally oriented dipoles is rather simple and has been previously solved. On the other hand, the case of vertically-oriented dipoles (i.e., aligned perpendicular to the layers) is more intricate, since the extracted terms cannot be transformed into well-known Green's functions. Previous works dealt with arrays of line and point sources, but did not address the critical task of computing the curl of the dyadic potentials, required to treat slot arrays and dielectric inclusions, whose available Floquet series expressions do not converge if the source and observation points lie in the same transverse plane.
\end{abstract}

Index Terms - Periodic problems, layered media, mixed potentials, Green's functions, Ewald method, leaky-wave antennas.

\section{INTRODUCTION}

$\mathrm{T}$ HE EFFICIENT computation of Green's functions in complex environments can extend the applicability of the method of moments (MoM) as an attractive approach to minimize the number of unknowns in electromagnetic problems. The case of vertically stratified media is a good example of a canonical geometry where Green's functions can be easily defined in a dyadic form [1]. While results are available for uniform structures, only recently an effort has been devoted to periodic Green's functions [2]-[6], required to study, e.g., planar stratified structures perturbed by arbitrarily shaped periodic loading (see Fig. 1). Computational issues associated with these potentials are addressed here.

When electric current sources are oriented horizontally (orthogonally to the stratification direction), only transverse potentials need to be computed. Asymptotic extractions can be used to improve the convergence of these slowly-converging series [2]. The extracted terms require the computation of homogeneous-medium periodic Green's functions for arrays of dipoles, whose accelerated numerical evaluation is achieved by standard means [7]. On the other hand, if an array of

Manuscript received April 1, 2015; revised May 15, 2015 and June 1, 2015; accepted July 1, 2015. Date of publication July 10, 2015; date of current version July 31,2015 . (Dates will be inserted by IEEE; "published"

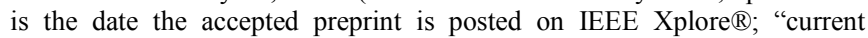
version" is the date the typeset version is posted on Xplore $\mathbb{R})$. Corresponding author: G.Valerio (e-mail: guido.valerio@upmc.fr). If some authors contributed equally, write here, "F. A. Author and S. B. Author contributed equally." IEEE TRANSACTIONS ON MAGNETICS discourages courtesy authorship; please use the Acknowledgment section to thank your colleagues for routine contributions.

Color versions of one or more of the figures in this paper are available online at http://ieeexplore.ieee.org.

Digital Object Identifier (inserted by IEEE).

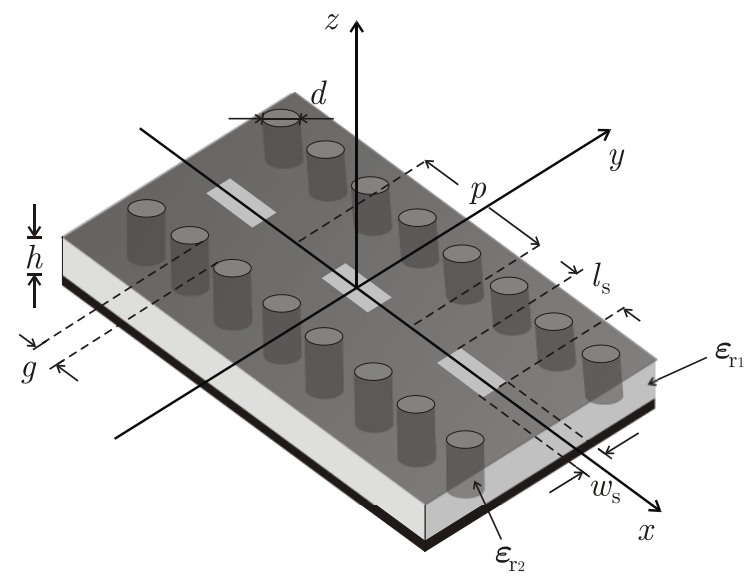

Fig. 1. Example of a structure requiring the computation of the vertical periodic potentials and the curls of dyadic periodic potentials: A substrate integrated waveguide structure synthesized by two rows of cylindrical dielectric inclusions in a host dielectric layer embedded between two metallic plates. Periodic slots are etched on the top metal plate.

vertical (parallel to $z$ ) electric dipoles is considered, vertical potentials are required; the extracted terms no longer correspond to arrays of dipoles, and standard computation techniques no longer hold.

In [5] and [6], a method has been recently proposed to transform these terms into modified periodic homogeneousmedium Green's functions, thus yielding a simple algorithm based on a modification of the Ewald method, which grants a remarkable convergence acceleration even in the case of complex modes and improper harmonics. This method is suitable for analyzing metallic objects using the EFIE. However, further potentials must be computed if the MFIE is used, or if periodic slots in PEC planes or periodic dielectric objects are included in the structure (see, e.g., Fig. 1). Specific methods are available to analyze similar structures if canonical 
shapes are present [8], but only a spatial-domain MoM provides a flexible handling of arbitrarily shaped objects. For these more general cases, integral formulations require the computation of both electric and magnetic fields due to both electric and magnetic equivalent currents. This is accomplished through the curls of the dyadic potentials, whose components are derivatives of the above-mentioned potentials. Due to Fourier-series properties, these potentials, arising from derivatives of the original ones, have a much slower spectral decay, thus posing serious computational problems. Their acceleration is thus necessary, especially for the case of onplane observation (source and observation points on the same transverse plane), where the original series expressions are actually divergent.

In this paper, we focus on the accelerated computation of periodic potentials in stratified media by means of suitable asymptotic extractions. In Section II we present the analytical formulation, while in Section III numerical results illustrating the accuracy and convergence of the method are discussed.

\section{EXTRACTION OF ASYMPTOTIC TERMS}

The four vertical potentials required to study general metallic objects in layered media (using the EFIE) are $P_{z}^{\mathrm{p}}$, $G_{A z x}^{\mathrm{p}}, G_{A z y}^{\mathrm{p}}$ and $G_{A z z}^{\mathrm{p}} \cdot G_{A z x}^{\mathrm{p}}$ and $G_{A z y}^{\mathrm{p}}$, are off-diagonal terms of the dyadic periodic potential $\underline{\mathbf{G}}_{A}^{\mathrm{p}}$, and can be suitably treated by differentiating the accelerated expressions obtained for $P_{z}^{\mathrm{p}}$. $G_{A z z}^{\mathrm{p}}$ can be treated similar to $G_{A x x}^{\mathrm{p}} \cdot P_{z}^{\mathrm{p}}$ is expressed as a series of integrals if a 1-D periodic array of dipoles is considered:

$$
\left.P_{z}^{\mathrm{p}}\left(\mathbf{r}, \mathbf{r}^{\prime}\right)=\frac{1}{2 \pi p} \sum_{n=-\infty}^{+\infty} e^{-j k_{x y} \Delta x} \int_{-\infty}^{+\infty} \tilde{i} \ldots\right) e^{-j k_{y} \Delta y} \mathrm{~d} k_{y},
$$

where $\tilde{i}$ is a 2-D spectral-domain Green's function, primed and unprimed coordinates refer to source and observation points, in the $l^{\prime}$ and $l$ layers, respectively. The period is $p, z$ is the stratification axis (see Fig. 1), $\Delta x=x-x^{\prime}, \Delta y=y-y^{\prime}$, and $k_{x n}$ $=k_{x 0}+2 \pi n / p$ the wavenumber of the $n$-th space harmonic, $k_{x 0}$ being the Bloch-mode wavenumber. As anticipated, both the series and the integrals are very slowly (algebraically) converging when the observation and source points lie on the same interface between layers. To enhance their convergence, the following asymptotic extraction of quasi-static terms can be performed:

$$
\begin{aligned}
& P_{z}^{\mathrm{p}}\left(\mathbf{r}, \mathbf{r}^{\prime}\right)=\frac{1}{2 \pi p} \sum_{n=-\infty}^{+\infty} e^{-j k_{x x n} \Delta x} \int_{-\infty}^{+\infty}[\tilde{I}, \ldots) \\
& \left.\begin{array}{lll}
\tilde{i} & & \prime^{\prime}
\end{array}\right] e^{-j k_{y} \Delta y} \mathrm{~d} k_{y}+P_{z}^{\mathrm{p}, \infty}\left(\mathbf{r}, \mathbf{r}^{\prime}\right) .
\end{aligned}
$$

The expressions for the extracted term $\tilde{i}$ can be found in [6], and will not repeated here for the sake of brevity. It is interesting to give the explicit expression of the closed-form solution for the spatial-domain term $P_{z}^{\mathrm{p}, \infty}$ : it is a sum of potentials $g^{\mathrm{p}, z}$ due to an array of half-line sources, computed by integrating the expressions for dipoles sources [6]. The integration can be performed analytically, resulting in

$$
\begin{gathered}
g^{\mathrm{p}, z}\left(\mathbf{r}, \mathbf{r}^{\prime}\right)=g_{\text {spectral }}^{\mathrm{p}, z}\left(\mathbf{r}, \mathbf{r}^{\prime}\right)+g_{\text {spatial }}^{\mathrm{p}, z}\left(\mathbf{r}, \mathbf{r}^{\prime}\right) \\
g_{\text {spectral }}^{\mathrm{p}, z}=\frac{1}{4 \sqrt{\pi} E p} \sum_{n=-\infty}^{+\infty} e^{-j k_{x x} \Delta x} \int_{1}^{\infty} \operatorname{erfc}\left(\frac{|\Delta z| E}{u}\right) e^{-\frac{\Delta y^{2} E^{2}}{u^{2}}+\frac{k_{\rho \rho}^{2} u^{2}}{4 E^{2}}} \mathrm{~d} u
\end{gathered}
$$

with $k_{\rho n}=\sqrt{k^{2}-k_{x n}^{2}}$ and $\Delta z=z-z^{\prime}$,

$$
g_{\text {spatial }}^{\mathrm{p}, z}=\frac{1}{4 \pi} \sum_{n=-\infty}^{+\infty} e^{-j n k_{x 0} p} \int_{E}^{\infty} \operatorname{erfc}(|\Delta z| s) \frac{e^{-R_{u n}^{2} s^{2}+\frac{k^{2}}{4 s^{2}}}}{s} \mathrm{~d} s
$$

with $R_{\mathrm{t} n}=\sqrt{(\Delta x-n p)^{2}+\Delta y^{2}}$, and $E$ is a suitable Ewald splitting parameter defined in [7, p. 254, (28), (31)-(34)].

The two series (4) and (5) have the same fast (Gaussian) convergence as the original Ewald series. Furthermore, the series converge even in the case of improper waves, i.e., waves not respecting the boundary conditions at infinity, often necessary to describe leaky waves [9]-[11].

The acceleration of the off-diagonal potentials $G_{A z x}^{\mathrm{p}}$ and $G_{A z y}^{\mathrm{p}}$ is then performed by extracting asymptotic terms proportional to $P_{z}$ through a $k_{x n}$ or $k_{y}$ factor, corresponding to $x$ - and $y$-derivatives of (4) and (5). These derivatives are easily computed analytically by differentiating each term of the sums individually, since the final series converges sufficiently fast (still Gaussian).

The magnetic field due to an electric current (needed in the MFIE) requires the curl of $\underline{\mathbf{G}}_{A}^{\mathrm{p}}$, and this is considered next. (The electric and magnetic fields due to magnetic currents, needed for slots and dielectric objects, may found from duality form the fields due to an electric current.) The nonzero terms of $\nabla \times \underline{\mathbf{G}}_{A}^{\mathrm{p}}$ can be expressed as

$$
\begin{gathered}
\left(\nabla \times \underline{\mathbf{G}}_{A}^{\mathrm{p}}\right)_{x x}=\partial_{y} G_{A z x}^{\mathrm{p}} \\
\left(\nabla \times \underline{\mathbf{G}}_{A}^{\mathrm{p}}\right)_{x y}=\partial_{y} G_{A z y}^{\mathrm{p}}-\partial_{z} G_{A y y}^{\mathrm{p}} \\
\left(\nabla \times \underline{\mathbf{G}}_{A}^{\mathrm{p}}\right)_{x z}=\partial_{y} G_{A z z}^{\mathrm{p}} \\
\left(\nabla \times \underline{\mathbf{G}}_{A}^{\mathrm{p}}\right)_{y x}=\partial_{z} G_{A x x}^{p}-\partial_{x} G_{A z x}^{p} \\
\left(\nabla \times \underline{\mathbf{G}}_{A}^{\mathrm{p}}\right)_{y y}=-\partial_{x} G_{A z y}^{p} \\
\left(\nabla \times \underline{\mathbf{G}}_{A}^{\mathrm{p}}\right)_{y z}=-\partial_{x} G_{A z z}^{p} \\
\left(\nabla \times \underline{\mathbf{G}}_{A}^{\mathrm{p}}\right)_{z x}=-\partial_{y} G_{A x x}^{p} \\
\left(\nabla \times \underline{\mathbf{G}}_{A}^{\mathrm{p}}\right)_{z y}=\partial_{x} G_{A y y}^{\mathrm{p}} .
\end{gathered}
$$

Their acceleration can still be performed as in (2), where the terms $\tilde{i}$ are replaced by suitable spectral terms: since we are dealing here with derivatives of the $\underline{\mathbf{G}}_{A}^{\mathrm{p}}$ components, these spectral terms will be proportional to the original $\underline{\mathbf{G}}_{A}$ terms with additional $k_{x n}$ and $k_{y}$ factors representing the $x$ - and $y$ derivatives, respectively. The presence of these terms further slows down the convergence of the unaccelerated potentials, and in some cases this leads to in-plane non-converging expressions. The relevant asymptotic behaviors for large 


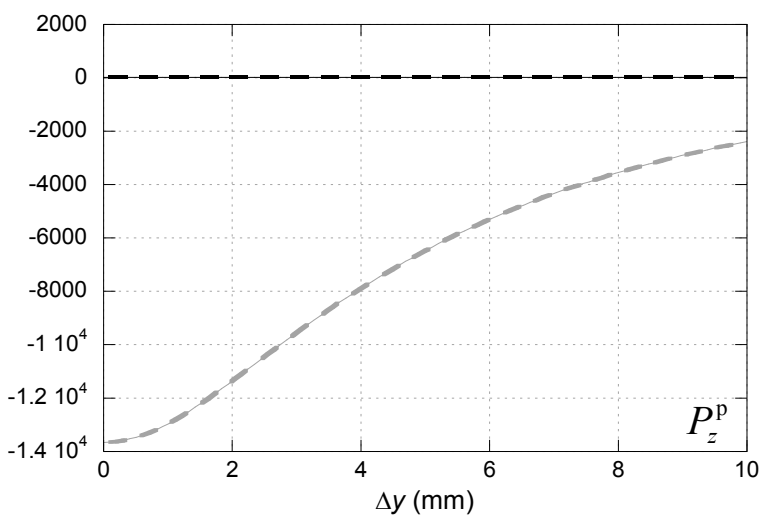

Fig. 2. Green's function $P_{z}^{\mathrm{p}}$ computed with (continuous lines) and without (dashed lines) acceleration: real parts (black lines) and imaginary parts (gray lines). The structure is a grounded slab in air, with $\varepsilon_{\mathrm{r} 1}=10.2, h=0.767 \mathrm{~mm}, p$ $=10 \mathrm{~mm}$, at a frequency $f=10 \mathrm{GHz}$. They are computed along the segment 0 $<\Delta y<p, \Delta x=p / 2$, and $z=z^{\prime}=0$ (air/substrate interface). The Bloch wavenumber is $k_{x 0}=1.5 k_{0}$ (bound mode, all the harmonics are proper [6], [11]). (Note: the terms are unitless.)

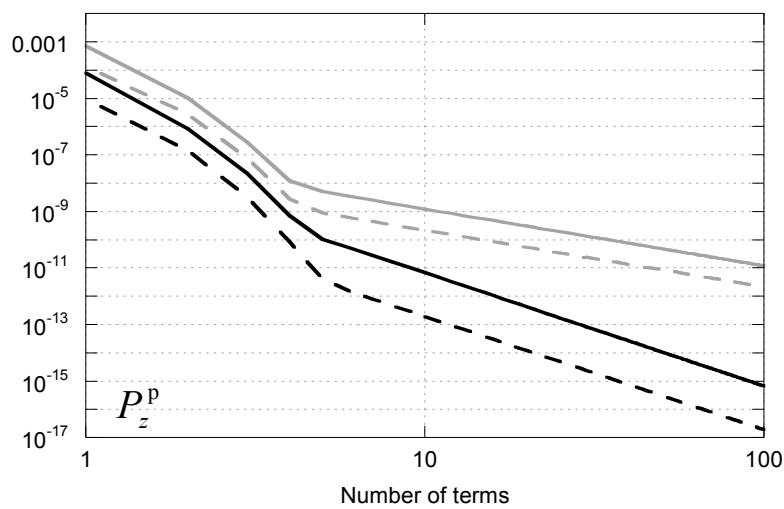

Fig. 3. Magnitude of the aggregated $(n,-n)$ terms of the unaccelerated (gray lines) and the accelerated (black lines) Green's function $P_{z}^{\mathrm{p}}$ vs. the summation index $n$. The structure is that of Fig. 2, but two periods are chosen: $p=10 \mathrm{~mm}$ (solid lines) and $p=4 \mathrm{~mm}$ (dashed lines). The Bloch wavenumber is $k_{x 0}=$ $(0.8-j 0.1) k_{0}$ (the $n=0$ harmonic is radiating and is improper [6], [11]). Coordinates: $z=z^{\prime}=0$ (air/substrate interface), $\Delta x=\Delta y=p / 2$.

values of $k_{x n}$ and $k_{y}$ can be derived once we recall those of the original spectral terms of $\underline{\mathbf{G}}_{A}^{\mathrm{p}}$ (always in the on-plane case):

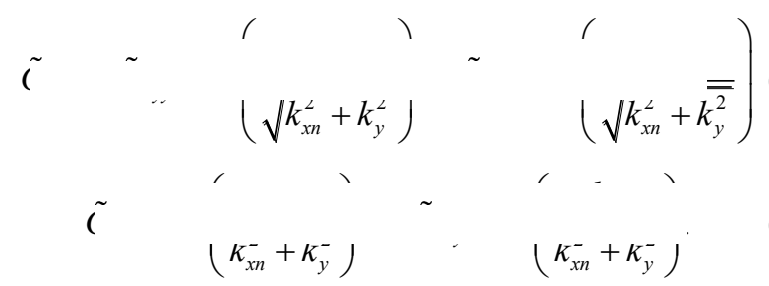

From (14) and (15), insufficient decay occurs for the series defining $\partial_{y} G_{A z x}^{\mathrm{p}}, \partial_{z} G_{A x x}^{\mathrm{p}}, \partial_{x} G_{A z x}^{\mathrm{p}}, \partial_{x} G_{A z z}^{\mathrm{p}}$, and $\partial_{x} G_{A y y}^{\mathrm{p}}$ in (6), (7), (9), (11), and (13), and for the integrals in each harmonic defining $\partial_{y} G_{A z z}^{\mathrm{p}}, \partial_{y} G_{A z y}^{\mathrm{p}}$, and $\partial_{y} G_{A x x}^{p}$ in (8), (10), and (12). These functions must be computed using extraction (2), since the original series (1) is no longer a valid representation. The second-order derivatives of (4) and (5) are then needed for the

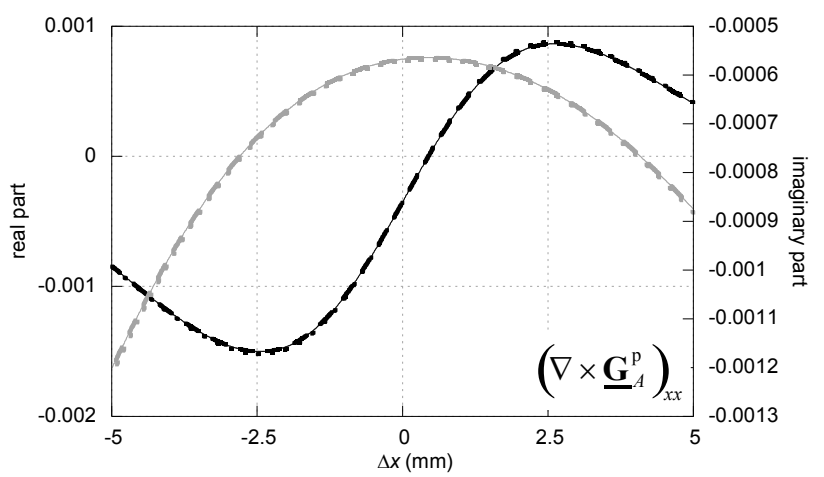

(a)

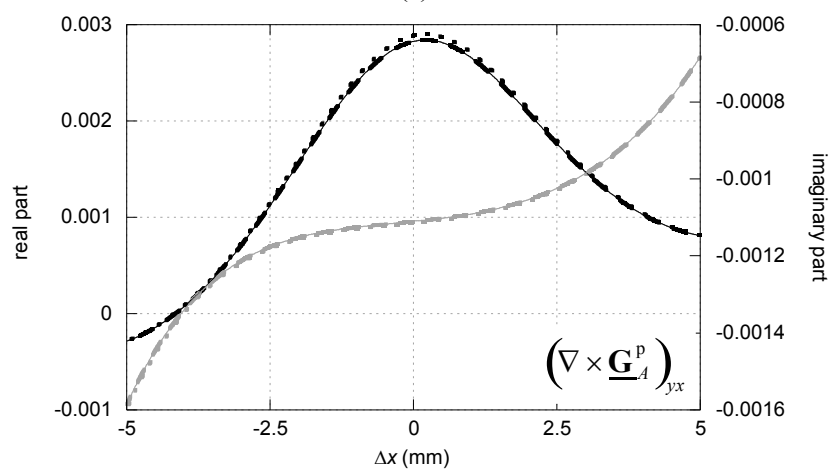

(b)

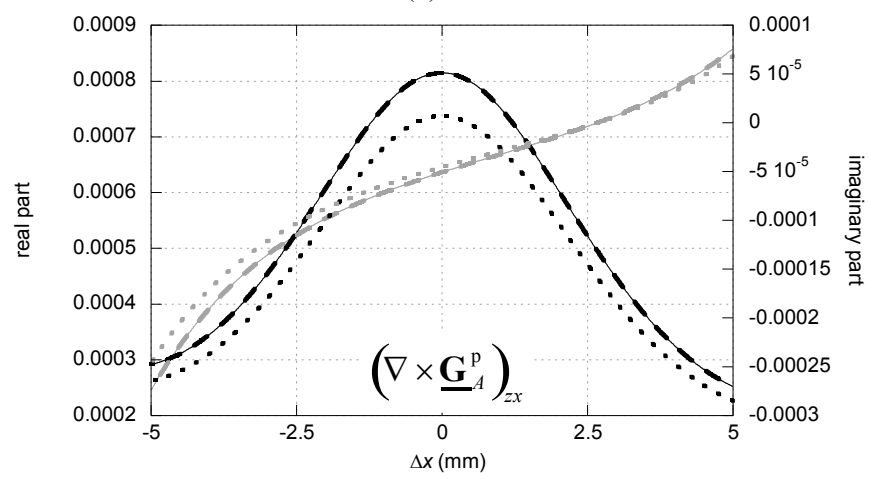

(c)

Fig. 4. Green's functions comparisons (the relevant potential is indicated in the figure): real parts (black lines) and imaginary parts (gray lines). The structure is that of Fig. 2. The potentials are computed along the segment $-p / 2$ $<\Delta x<p / 2, \Delta y=p / 2$, and $z^{\prime}=0$. The Bloch wavenumber is $k_{x 0}=-0.5 k_{0}-j 0.2 k_{0}$ (the $n=0$ harmonic is radiating and is proper [6], [11]). Potentials computed with (continuous lines) and without (dashed lines) acceleration at $z=0.1 \mathrm{~mm}$ (off plane) and with acceleration (dotted lines) at $z=0$ (on plane). (Note: the terms plotted are unitless.)

spatial part. As anticipated, they can be computed analytically by differentiating the series term by term; the results are not reported here for the sake of brevity.

\section{NUMERICAL RESULTS}

In Fig. 2, we show the comparison between the unaccelerated and the accelerated Green's function $P_{z}^{\mathrm{p}}$ along the segment $0<\Delta y<p, \Delta x=p / 2, z=z^{\prime}=0$ (at the interface between the slab and the air, i.e., in-plane case). In both cases the expressions are convergent. The agreement between the curves verifies the correctness of the acceleration procedure for this term. 


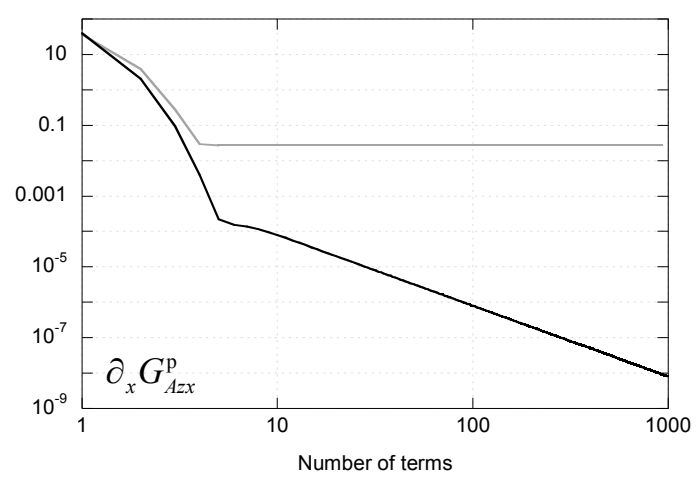

(a)

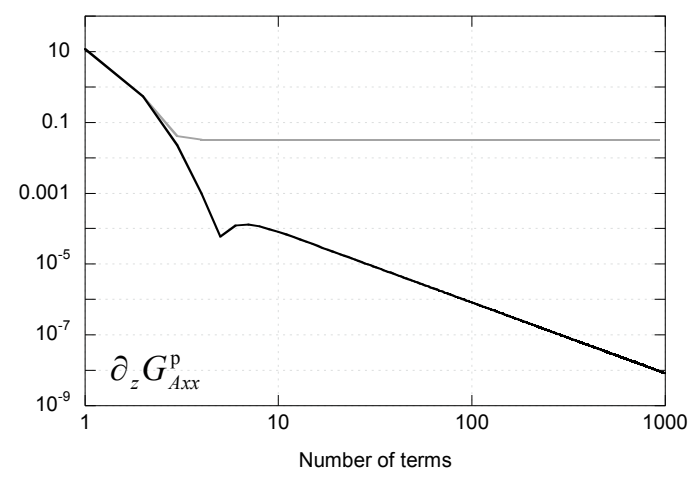

(b)

Fig. 5. Magnitude of the aggregated $(n,-n)$ terms of the unaccelerated (gray lines) and the accelerated (black lines) derivatives of the dyadic potential components (the relevant potential is indicated in the figure), vs. the summation index $n$. The structure is that of Fig. 2. The Bloch wavenumber is $k_{x 0}=-0.5 k_{0}-j 0.2 k_{0}$ (the $n=0$ harmonic is radiating and is proper [6], [11]). Coordinates: $z=z^{\prime}=0$ (air/substrate interface), $\Delta x=\Delta y=p / 2$.

In Fig. 3, we show the decay of the terms in the series for two values of the spatial period $p$, with and without the extraction of $\tilde{i}$. The enhanced convergence rate is clearly visible by analyzing the slope of the curves (from $n^{-2}$ to $n^{-4}$ ).

In Fig. 4(a), (b), and (c), we show the behaviors of the terms in (6), (9), and (12), respectively, along the line segment defined by $0<\Delta x<p, \Delta y=p / 2$. In particular, comparison between the unaccelerated and the accelerated Green's functions at $z^{\prime}=0 \mathrm{~mm}$ and $z=0.1 \mathrm{~mm}$ (i.e., off-plane case) are shown. The off-plane case has been chosen here since both the expressions are convergent, thus providing partial validation of the acceleration procedure (note the excellent agreement between the continuous and dashed curves). The on-plane case at the air/dielectric interface is also shown, but only for the accelerated Green's functions, since the unaccelerated expression is not convergent for the three components chosen. The computation of the in-plane case cannot be simply performed by replacing $z=0$ with a small displacement along $z$ for two main reasons. First, the sensitivity with respect to $z$ is difficult to predict (see, e.g., the good agreement in Figs. 4(a), (b) and the worse agreement in Fig. 4(c)). Second, even by keeping a small displacement along the $z$ axis, when computing 1000 samples on the line described in the caption of Fig. 4, the convergence of each unaccelerated sum requires about 50 terms for a relative error of $10^{-4}$, as compared to about 4 terms for the accelerated series, equivalent to $162.2 \mathrm{vs.}$ 19.1 seconds, on an Intel i7-3930K CPU@3.20 GHz. The acceleration obtained is extremely relevant also for small values of $z-z^{\prime}$. In Fig. 5, we show the decay of the terms in the series for the derivative components $\partial_{x} G_{A z x}^{\mathrm{p}}$ and $\partial_{z} G_{A x x}^{p}$, both used in (9), with and without extractions, for the structure in Fig. 4 at the air/dielectric interface (i.e., $z=z^{\prime}=0$ ). It is observed that the asymptotic behavior of the unaccelerated terms is a constant, thus making the convergence of (9) impossible. Analysis of the slope of the relevant curves reveals a convergence rate of $n^{-2}$ for the accelerated expressions.

\section{CONCLUSION}

We have proposed asymptotic extractions for periodic potentials due to vertical currents in layered media. Furthermore, the computation of the curls of the dyadic potentials, required in the analysis of slot arrays and dielectric inclusions, has been addressed in detail. Numerical results have demonstrated the accuracy of the acceleration schemes and the enhanced convergence of the spectral series.

\section{REFERENCES}

[1] K. A. Michalski and D. Zheng, "Electromagnetic scattering by sources of arbitrary shape in layered media, Part I: Theory," IEEE Trans. Antennas Propag., vol. 38, no. 3, pp. 335-344, Mar. 1990.

[2] G. Valerio, P. Baccarelli, S. Paulotto, F. Frezza, and A. Galli, "Regularization of mixed-potential layered-media Green's functions for efficient interpolation procedures in planar periodic structures," IEEE Trans. Antennas Propag., vol. 57, no. 1, pp. 122-134, Jan. 2009.

[3] A. L. Fructos, R. R. Boix, and F. Mesa, "Efficient computation of the off-diagonal elements of the vector-potential multilayered periodic dyadic Green's function," IEEE Trans. Antennas Propag., vol. 59, no. 7, pp. 2557-2564, Jul. 2011.

[4] D. Van Orden and V. Lomakin, "Rapidly convergent representations for periodic Green's functions of a linear array in layered media," IEEE Trans. Antennas Propag., vol. 60, no. 2, pp. 870-879, Feb. 2012.

[5] G. Valerio, D. R. Wilton, D. R. Jackson, and A. Galli, "Acceleration of mixed potentials from vertical currents in layered media for 2-D structures with 1-D periodicity," IEEE Trans. Antennas Propag., vol. 60, no. 8, pp. 3782-3793, Aug. 2012.

[6] G. Valerio, P. Paulotto, P. Baccarelli, D. R. Jackson, D. R. Wilton, W. A. Johnson, and A. Galli, "Efficient computation of 1-D periodic layered mixed potentials for the analysis of leaky-wave antennas with vertical elements," IEEE Trans. Antennas Propag., vol. 63, no. 6, pp. 2396-2411, Jun. 2015.

[7] F. Capolino, D. R. Wilton, and W. A. Johnson, "Efficient computation of the 3D Green's function for the Helmholtz operator for a linear array of point sources using the Ewald method," J. Comp. Phys., vol. 223, no. 1, pp. 250-261, Apr. 2007.

[8] M. Casaletti, G. Valerio, J. Seljan, M. Ettorre, and R. Sauleau, "A fullwave hybrid method for the analysis of multilayered SIW-based antennas," IEEE Trans. Antennas. Propag., vol. 61, no. 11, pp. $5575-$ 5588, Nov. 2013.

[9] A. A. Oliner and D. R. Jackson, "Leaky-wave antennas," in Antenna Engineering Handbook. J. L. Volakis, Ed. New York, NY: McGrawHill, 2007, ch. 11.

[10] P. Baccarelli, C. Di Nallo, S. Paulotto, and D. R. Jackson, "A full-wave numerical approach for modal analysis of $1 \mathrm{D}$ periodic microstrip structures," IEEE Trans. Microw. Theory Tech., vol. 54, pp. 1350-1362, Apr. 2006.

[11] P. Baccarelli, S. Paulotto, D. R. Jackson, and A. A. Oliner, "A new Brillouin dispersion diagram for 1-D periodic printed structures," IEEE Trans. Microw. Theory Tech., vol. 55, pp. 1484-1495, Jul. 2007. 